dust and endotoxin exposure. Decreased dust exposures were seen when cleaning of milking areas or reparation of buildings was performed. Robotic milking was associated with increased dust levels when compared to parlour milking.

Conclusion These initial findings provide information on working tasks that determine the level of personal exposure to dust and endotoxin during dairy farming. By June 2013, the authors intend to present results from statistical models which will examine the combined effects of farm characteristics and working tasks.

\section{OCCUPATIONAL EXPOSURES IN VETERINARIANS: FINDINGS FROM A NATIONAL SURVEILLANCE PROJECT (CAREX CANADA)}

${ }^{1} \mathrm{~L}$ Hall, ${ }^{1}$ Peters, ${ }^{1}$ Davies, ${ }^{2}$ Demers. ${ }^{1}$ University of British Columbia, Vancouver, Canada; ${ }^{2}$ Occupational Cancer Research Centre, Cancer Care Ontario, Toronto, Canada

\subsection{6/oemed-2013-101717.319}

Objectives Veterinarians work in a variety of environments with diverse patient types. Risk of physical injury and zoonoses are common concerns for this group, but other exposures may also produce adverse health outcomes. The objective of this study was to identify Canadian veterinary exposure prevalence and levels for ionising radiation (IR) and antineoplastic agents (AAs), as part of the CAREX Canada project.

Methods For IR, we used 2008 whole-body dose data from a national government exposure registry. Veterinarians with measured levels of exposure were identified. The proportions exposed were combined with 2011 national veterinary statistics to estimate the prevalence range within two exposure categories. For AAs, prevalence and exposure category estimates were formed by combining the 2011 national veterinary statistics with information on practice type and AA usage rates obtained from provincial veterinary associations, peer-reviewed literature, and veterinary field experts.

Results In 2008, 26\% of Canadian veterinarians were monitored for IR exposure. Of the 3,155 veterinarians monitored, 282 (8.9\%) had a dose $>0 \mathrm{mSv}$. Extrapolating to all veterinarians in Canada, we estimate a maximum of 1070 are exposed to IR doses above zero $\mathrm{mSv}$. The majority $(\mathrm{n}=278-1055)$ fall within a low dose category $(>0-1 \mathrm{mSv})$ while $n=4-15$ are exposed to levels between 1-5 mSv. None had doses $>5 \mathrm{mSv}$. We estimate that $18 \%$ of veterinarians $(n=2,200)$ are exposed to AAs; these fall into two categories of moderate exposure, defined as "low frequency, low control" ( $\mathrm{n}=2,180)$ and "high frequency, high control" $(\mathrm{n}=20)$.

Conclusions CAREX Canada's exposure estimates could be used to assist in the development of epidemiological studies or risk assessments. Our estimates indicate that exposure to IR and AAs in veterinarians is low, however the accuracy of our findings is limited by data sources of varying quality. We plan to refine our current estimates and assess other exposures in veterinary settings.

\section{EXPOSURE ASSESSMENT OF THORACIC AEROSOL IN AN INTERNATIONAL PROSPECTIVE STUDY OF CEMENT PRODUCTION WORKERS}

H N Notø, Eduard. National Institute of Occupational Health, Oslo, Norway

10.1136/oemed-2013-101717.320
Hilde Notø, Karl-Christian Nordby, ['ivind Skare, Helge Kjuus and Wijnand Eduard

Background and Objectives Respiratory effects have been linked to aerosol exposure in cement production workers. This presentation aims at estimating annual exposure levels to thoracic aerosol during the study period. The thoracic fraction was chosen because dynamic lung function was the main outcome of the international study.

Methods We collected 7120 personal shift measurements of thoracic aerosol contributed by 2866 persons within 8 job types in 24 plants in 2007, 2009 and 2011. Measurements above 150 $\mathrm{mg} / \mathrm{m}^{3}$ were excluded as light microscope analysis revealed larger particles than expected from the thoracic convention $(\mathrm{N}=63)$. Measurements with absolute Z-scores above 3.29 in models including job type, plant and year were also excluded $(\mathrm{N}=44)$, as well as samples with technical errors $(\mathrm{N}=71)$. Arithmetic mean (AM) exposures were estimated using mixed regression modelling of the ln-transformed exposure. The final model included plant, job type, plant*job type, year, plant*year and season as fixed effects, individuals as random effect, and plantspecific residuals.

Results Of the workers $86 \%$ had been measured more than once, on average 2.4 times. All fixed factors contributed significantly to the models. Plant specific residuals also improved the model significantly. A job exposure matrix was constructed for plant-specific job types for each year averaged across seasons. AM exposure levels were estimated by exponentiation of the sum of the regression coefficients of the fixed effects and the half of the plant-specific residual variances. The estimated exposure varied between job types and plants from 0.13 to $14 \mathrm{mg} / \mathrm{m}^{3}$.

\section{OCCUPATIONAL EXPOSURES TO KNOWN AND SUSPECTED CARCINOGENS IN THE CANADIAN CONSTRUCTION INDUSTRY}

${ }^{1} \mathrm{~B}$ Ge, ${ }^{1}$ Peters, ${ }^{2}$ Demers. 'University of British Columbia, Vancouver, Canada; ${ }^{2}$ Occupational Cancer Research Centre, Toronto, Canada

\subsection{6/oemed-2013-101717.321}

Objectives CAREX Canada aims to estimate the number of Canadian workers exposed to various carcinogens in the workplace. The objectives of this work is to determine the number of workers exposed to different carcinogens in the construction industry in Canada and identify high risk occupations within the industry for exposure.

Methods Data from the Canadian Workplace Exposure Database (CWED), published relevant Canadian and US scientific literature, data from previous European CAREX projects, government grey literature and other technical reports were used to develop exposure proportions for each occupation in the construction industry. These proportions are combined with 2006 Canadian census of population data to obtain the prevalence of exposure for 30 carcinogens selected to be relevant in the Canadian context.

Results Canadian construction workers, with a total population of 1.07 million, are estimated to have over $1,188,000$ exposures to the 30 selected carcinogens. Some workers are likely exposed to more than one substance at a time. Carcinogens with substantial number of workers exposed include: solar ultraviolet radiation (343,000 workers exposed), crystalline silica $(240,000$ exposed), wood dust (166,000 exposed), asbestos (134,000 exposed), diesel engine exhaust (84,000 exposed), lead and lead compounds (51,000 exposed) and bitumen (50,000 exposed). 
Jobs at high risk of exposure include construction trade helpers and labourers $(290,000$ exposures), carpenters $(227,000$ exposures), and heavy equipment operators $(127,000)$. Quantitative exposure level estimates are available for some carcinogens.

Conclusions Safety in the construction industry has long been the focus of scientific research. Our work shows that workers in this industry are also exposed to a number of known and suspected human carcinogens, with some exposures being very prevalent currently in Canada. Results from our study may be used by occupational exposure and epidemiologic studies to further investigate exposures and occupational cancer in this unique industry.

\section{Session: 19. Life course trajectories}

\section{UNEMPLOYMENT AT A YOUNG AGE AND LATER UNEMPLOYMENT IN NATIVE SWEDISH AND IMMIGRANT YOUNG ADULTS}

M Helgesson, B J Johansson, T N Nordquist, I L Lundberg, E V Vingård. Uppsala University, Uppsasa, Sweden

\subsection{6/oemed-2013-101717.322}

Objectives The youth unemployment rate is increasing around the world due to recent recessions in the worldwide economy. Unemployment has been seen to affect future work participation. This register-based study examined the long-term effect of unemployment on future unemployment in Sweden.

Methods The study group of 199,623 individuals included all immigrants born between 1968 and 1972 who immigrated to Sweden before $1990(25,607)$ and a random sample of native Swedes of the same age $(174,016)$. The follow-up period was 15 years, from 1993 to 2007.

Results Individuals who were unemployed in 1992 had an elevated risk of $\geq 100$ days of unemployment during the whole follow-up period. The risk of unemployment in the follow-up increased with the length of unemployment in 1992. Immigrants had a higher risk of unemployment both at baseline and followup compared with native Swedes, but followed the same pattern as native Swedes when exposed to unemployment. For individuals with $\geq 100$ days of unemployment in 1992, participation in an Active Labour Market Programme (ALMP) increased the risk of future unemployment, whereas both higher educational level at baseline and attainment of a higher educational level between 1992 and 1997 decreased the risk of future unemployment.

Conclusion Exposure to unemployment was associated with elevated risk of future unemployment 15 years after exposure. In addition to the human suffering caused, this can mean substantial costs in the form of increased welfare payments and loss of productivity and tax income to a society. ALMPs seem ineffective in promoting future work participation.

\section{SELF-RATED HEALTH BEFORE AND AFTER EMPLOYMENT TRANSITIONS: EVIDENCE IN EUROPEAN COUNTRIES}

M Schuring, Burdorf. Erasmus MC, Rotterdam, The Netherlands

\subsection{6/oemed-2013-101717.323}

Objectives The aim of this study was to investigate self-rated health before and after labour force exit due to unemployment, economic inactivity or early retirement. A secondary objective was to investigate health before and after entering paid employment

Methods Trajectories of self-rated health in 520.830 employed and 278.954 non-employed persons were examined from the European Community Household Panel Survey (ECHP) for up to 5 years before and 6 years after labour force exit or reemployment, with yearly measurements from 1994-2001. Data were analysed by use of repeated-measures logistic regression with generalised estimating equations.

Results The likelihood of poor health increased among persons who became unemployed $(\mathrm{OR}=1.15,95 \%$ CI 1.04-1.28) or economically inactive $(\mathrm{OR}=1.29$ 95\% CI 1.17-1.42). Among persons who left the labour force due to early retirement the likelihood of poor health increased in the years before retirement $(\mathrm{OR}=1.1095 \%$ CI 1.07-1.13), whereas this increase was less steep in the years after early retirement. Among unemployed persons who re-entered paid employment the likelihood of poor health decreased $(\mathrm{OR}=0.8095 \%$ CI 0.71-0.91).

Conclusions Health is influenced by employment transitions into and out of the labour force. Policies should protect persons who leave the labour force against further deterioration of health. Entering paid employment is an important measure to improve self-rated health among unemployed persons.

\section{LABOUR MARKET TRAJECTORIES AND PERMANENT DISABILITY. STUDY OF PROGNOSTIC FACTORS}

X. Duran Jordà, Benavides, Martínez Martínez. CiSAL- Universitat Pompeu Fabra, Barcelona, Spain

\subsection{6/oemed-2013-101717.324}

Objectives In the current context of increasing population ageing and after trends of labour market flexibilisation, this study analyses the impact of labour trajectories on the potential years of working life lost (PYWLL) due to non-work related permanent disability (PD).

Methods Retrospective cohort of 14,999 workers affiliated with the Social Security System in Spain that began a non-work related PD between 2004 and 2010. The PYWLL is defined as the time in years between the age at which a worker initiates a PD and the official retirement age (65 years) or the age of reinstatement to a job. Two indexes of labour trajectory were obtained by Principal Components Analysis: Index 1 (number of contracts, number of unemployment periods and number of periods without affiliation) and Index 2 (percentage of inactive time). Median differences in PYWLL (MD) and 95\% confidence intervals (CI95\%) were computed using a median regression. The main independent variable was obtained combining tertiles (low, medium, high) of index 1 and 2 ((Index 1, Index 2): (high, high), (high, low), [3DOTS], (low, low)) with category (low, low) as reference. Analysis were stratified by gender and adjusted by the total time elapsed by each worker in the labour trajectory.

Results The median PYWLL was 8.7 for men and 11.0 for women. The greatest crude MD was for category (high, high) for men (MD:4.70, CI95\%:3.88, 5.52) and (medium, high) for women (MD:4.09, CI95\%:2.93, 5.25). The greatest adjusted difference in medians was for category (high, high) in men (MD:3.05, CI95\%:2.64, 3.47) and in women (MD:3.68, CI95\%:2.57, 4.68).

Conclusion Workers with a labour trajectory corresponding to the highest indexes have the greatest loss of PYWLL due to nonwork related PD. Greater labour market flexibilisation could 